\title{
Intrinsic mode functions and fractal characteristics of the solar Incoming Shortwave Radiation observed at Nalohou (Benin Republic)
}

\section{Koto N'Gobi Gabin ${ }^{1,}$, Agbazo Medard ${ }^{1,2}$, Mamadou Ossenatou ${ }^{1,3}$, Togbenou Koffi ${ }^{4}$ and Kounouhewa Basile ${ }^{1}$}

\author{
${ }^{1}$ Laboratoire de Physique du Rayonnement (LPR), UAC, BP : 526 UAC Benin \\ ${ }^{2}$ International Chair in Physics Mathematics and Applications (CIPMA-Chair UNESCO), BP: 526 UAC Benin \\ ${ }^{3}$ Institut de Mathématiques et de Sciences Physiques, BP : 613, Porto-Novo, Benin \\ ${ }^{4}$ China Railway Major Bridge Reconnaissance and Design Group Co., Ltd., Wuhan, 430056, China \\ Corresponding author email: kotgabin36@yahoo.fr
}

\section{INFOS SUR L'A R T I C L E}

\section{Historique de l'article:}

Reçu le : 31 juillet 2019

Reçu en format révisé le : 09 novembre 2019

Accepté le : 13 novembre 2019

Mots-Clés: DME-AMFST, FMIO, rayonnement solaire, analyse multifractale, decomposition

Keywords: EMD-MFDFA, IMF, solar radiation, multifractal analysis, decomposition

\begin{abstract}
RE S U M E
L'énergie solaire est une énergie propre et renouvelable provenant du rayonnement solaire. Cependant, la quantité de Rayonnement Solaire Incident (RSI) parvenant au sol dépend des conditions météorologiques (exemple la couverture nuageuse), ce qui la rend intermittente, aléatoire et non-linéaire. Ces propriétés complexes conduisent à une grande fluctuation du rayonnement solaire disponible. Dans ce travail, nous avons étudié les propriétés fractales et déterminé les oscillations intrinsèques du RSI en utilisant simultanément les méthodes de : (a) Décomposition Modale Empirique (DME), (b) l'analyse multifractale du spectre (MFS) de RSI et (c) l'Analyse Multifractale de la Fluctuation sans Tendance (AMFST). Les données de RSI au pas de temps de la $1 / 2$ heure, mesurées en 2008 et 2009 à la station AMMA-CATCH de Nalohou (Bénin) sont utilisées. Les résultats révèlent que la série temporelle peut être décomposée en douze (12) Fonctions Modales Intrinsèques Oscillatoires (FMIO) et une tendance polynomiale décroissante. La série du RSI n'est pas un bruit blanc donc peut être prédite. Les propriétés multifractales obtenues varient d'uneFMIO à l'autre. De plus, il existe une disparité entre les propriétés multifractales de chacune des douze FMIO et celles de la série originale. Ainsi, aucune des douze FMIO ne peut à elle seule représenter la série originale et la force de leurs multiracialités ne dépend pas des périodes d'oscillation des FMIO.
\end{abstract}

\begin{abstract}
Solar energy is clean and renewable energy obtained from solar radiation. However, the quantity of the Incoming Shortwave Radiation (ISR) reaching the ground depends on meteorological conditions (e.g. clouds covers) which make it intermittent, randomness and nonlinear. These complex properties lead to strong fluctuation of the available solar radiation power. In this work, we have studied fractal properties and determine the intrinsic oscillations of ISR using simultaneously the methods of: (a) Empirical Mode Decomposition (EMD), (b) Multifractal Spectra (MFS) analysis and (c) Multifractal Detrended Fluctuation Analysis (MFDFA). Data of ISR recorded at $1 / 2$ hour time scale, measured in 2008 and 2009 at Nalohou AMMA-CATCH Station (Benin) are used. The results show that ISR time series can be decomposed into twelve (12) oscillatory Intrinsic Mode Functions (IMFs) with a decreasing polynomial trend. ISR is not a white noise and can be predicted. The multifractals properties obtained vary from oneIMF to another. Moreover, there is disparity between multifractal properties of each IMF and those of the original time series. So, none of the IMFs can represent the original time series and the strength of their multifractality does not depend on the oscillation period of the IMFs.
\end{abstract}




\section{INTRODUCTION}

West Africa is one of the sunniest areas of the world (Chu, 2011) where solar energy can be exploited as huge potential of renewable and clean energy, useful for electricity. Benin Republic is one of the West African countries where electrification is improving gradually due to the transformation of solar energy into electricity using photovoltaic (PV) technologies. The PV plants efficiency depends greatly on the solar ISR (Zeng et al., 2013). In addition, ISR is one of the essential climate variables, important for understanding climate evolution (Bengulescu et al., 2016). However, ISR data are complex and have relationship with geographical location, as well as local meteorological factors, such asair temperature, water vapor, suspended solids, and cloud cover (Dos Anjos et al., 2015). These meteorological factors constitute a strongly coupled multi-variable nonlinear system and ISR is just an output variable of this system. Thus, ISR has intermittence, randomness, uncertainty, nonlinearity and nonstationary which may cause power fluctuations of utility grid and the voltage instability at the point of common coupling (Hoff et al., 2007). These stochastic characteristics of ISR lead to large amplitude and frequent fluctuation of solar power, which make power prediction difficult. Meanwhile, for better power dispatch, it is necessary to improve power generation of PV plants, depending on the prediction of solar radiation. Literature shows that a good knowledge of ISR variations and its internal mechanisms (nonlinear and nonstationary characteristics) are necessary foran efficient prediction and to perform numerical analysis (Calif et al., 2013; Zeng et al., 2013).

Several methods from literature are employed to analyze measured meteorological data in general and natural phenomenon; among these methods one can cite neural networks (Hocaoglu et al., 2008; Behrang et al., 2010), s-transformation (Zhang et al., 2014), Singular Value Decomposition (SVD) (Berger et al.,1997) and Markov chains (Mellit et al., 2005). But, these methods of data modeling ignore the internal mechanisms driving meteorological data such as ISR.

Wavelets methods are the most popular methods used in literature (Sang, 2012), but their capabilities are constrained by the nonlinearity of the data sets, the selection of appropriate mother wavelet function and the selection of appropriate levels of decomposition. As an alternative to Wavelets, a fully data adaptive multiscale decomposition method namely known as Empirical Mode Decomposition (EMD) was proposed by Huang et al. (1998). One of the main differences between EMD and Wavelets is the choice of a time invariant filter (wavelet function) made in Wavelets. This choice is not generally suited to local variations, unlike EMD (Flandrin and Gonçalvès, 2004). EMD is robust in the presence of nonlinear and nonstationary data. Therefore, EMD is a useful and objective method for studying time series (Huang et al., 1998; 2003; Wu and Huang, 2003).

Furthermore, Fractal theory introduced by Mandelbrot (1982) is one of the most useful methods for complex natural phenomenon time series analysis, especially tocharacterize its high variability (Mandelbrot, 1982; Schertzer and Lovejoy, 1987, Calif et al., 2013).

Multifractal Detrended Fluctuation Analysis (MFDFA) method developed by (Kantelhardt et al., 2002 ) is the most popular fractal method used. For example, Zeng and al. (2013) in North America have studied the nonlinear characteristics of solar radiation by MFDFA method. The finding permits PV plant location section, their capacity determination and solar radiation prediction. In Benin Republic, some excellent work had explored surface radiation budget in Sudanian climate (Kounouhewa et al., 2013; Mamadou et al., 2014). However, the nonlinear and nonstationary characteristics of the observed ISR have not been sufficiently documented. This study aims to overcome this lack by combining two physical approaches namely the EMD and MFDFA, in order to highlight the (1) oscillation modes and (2) characterize the random behavior of the ISR which are embedded in them.

The paper is organized as follows: in section 2, the details of the incoming shortwave radiation time series are provided; EMD, MFDFA and the Multifractal Spectra method are briefly described. In section 3 , we present the results and analysis. The conclusions follow in section 4 .

\section{MATERIALS AND METHODS}

\subsection{Materials}

\subsubsection{Site Description}

The studied site, Nalohou station $\left(9^{\circ} 74^{\prime} 48 \mathrm{~N}\right.$, $1^{\circ} 60^{\prime} 46 \mathrm{E}$, a.s.1 449m) is in the Donga catchment (Djougou, Northern Benin). The station is one of the African Monson Multidisciplinary Analysis (AMMA) measurements site (See Mamadou et al., 2014), installed since July 2007 (Figure 1). The measurement site is a cultivated area, located in a Sudanian climate characterized by a long term (1959-2005) mean annual precipitation of $1190 \mathrm{~mm} /$ year, a mean air temperature is $\sim 29^{\circ} \mathrm{C}$ and the mean Relative Humidity is $\sim 80 \%$. The rainfall regime in the region is driven by the seasonal migration of the Intertropical Convergence Zone (ITCZ) (Lélé et al., 2010). Sudanian climate is characterized by a single dry season, often starting from October or November to February or March and a single wet season from April to October. Several meteorological parameters, radiative energy and heat surface fluxes are measured for understanding the Western African Monsoon dynamics (Mamadou et al., 2014). For this study we used data collected by a kipp and Zonen CRN1 Pyranometer, which has measured and recorded the ISR 
in a data logger (CR 3000) with a time scale of 30 minutes.

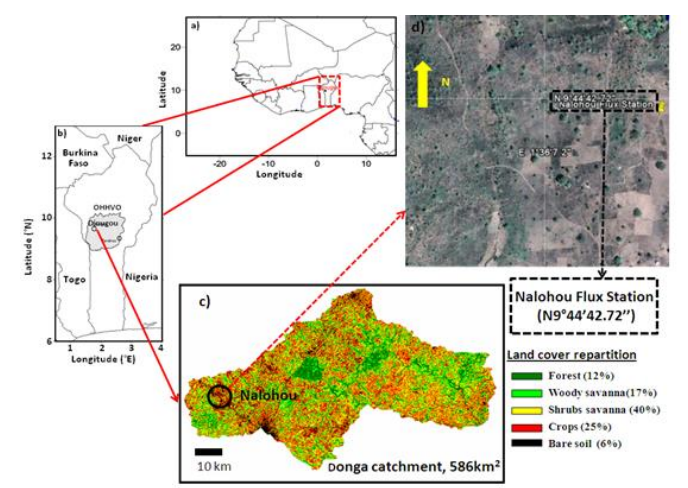

Fig. 1: Location of Nalohou station: (a) Location of Benin in Africa; (b) Location of Djougou in Benin; (c) Land cover of the Donga catchment; (d) Nalohou station location

\subsubsection{Data description}

Figure 2 presents the box plot statistics of the mean value of the daily ISR recorded between 2008 and 2009. The maximum, median and minimum daily values are respectively around 320,250 and $10 \mathrm{~W} / \mathrm{m}^{2}$. The shape of the boxplots shows that ISR data are asymmetric, therefore, they are not distributed according to the normal law.

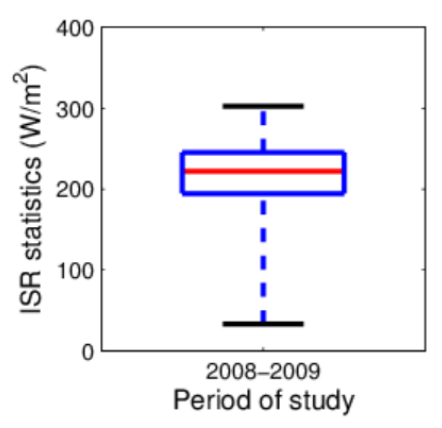

Fig.2: Box plot statistics of incoming shortwave radiation

\subsection{Methods}

\subsubsection{Empirical Mode Decomposition (EMD)}

According to (Huang et al., 1998; Huang, 2005), the basic idea of EMD is to decompose a complicated signal into a finite and a small number of oscillatory modes based on a local characteristic time scale. Each oscillatory mode is expressed by an intrinsic mode function (IMF), which has to satisfy the two conditions as follows: (1) In the whole dataset, the number of extrema and the number of zero-crossings must either be equal or differ at most by one; (2) At any point, the mean between the upper and lower envelopes must be zero. With the definition of the
IMFs, one can then decompose any function $x(t)$ through a sifting process:

Let $\left\{x_{t} \mid t=1,2, \ldots, N\right\}$ be a given ISR times series, the computational steps of the sifting process are described as follows: (1) Identify all the local extrema (minima and maxima) of $x(t)$ and connect all the local maxima and minima using a cubic spline to generate an upper envelope $\mathrm{u}(\mathrm{t})$ and a lower envelope $v(t)$. (2) Calculate the mean envelop $m(t)$ by averaging the upper envelope $u(t)$ and the lower envelope $v(t)$, and then deduce a detailed component $d(t)=x(t)-m(t)$. (3) Check whether $d(t)$ is an IMF. If $d(t)$ is an IMF, then set $c(t)=d(t)$ and meanwhile replace $x(t)$ with the residual $r(t)=x(t)-c(t)$. Otherwise, replace $x(t)$ with $d(t)$ and repeat Steps 1-2, until the following termination criterion is satisfied:

$\sum_{t=1}^{N} \frac{\left[d_{j-1}(t)-d_{j}(t)\right]^{2}}{\left[d_{j-1}(t)\right]^{2}} \leq \delta$

Where $\mathrm{j}$ denotes the number of iterative calculations. The threshold value $\delta$ was set to 0.2 in this study. (4) Repeat Steps 1-3, until all the IMFs and residual are obtained. Finally, the original time series $x(t)$ can be decomposed as follows:

$\mathrm{x}(\mathrm{t})=\sum_{\mathrm{i}} \mathrm{c}_{\mathrm{i}}(\mathrm{t})+\mathrm{r}(\mathrm{t})$

Where $c_{i}(t)$ and $r(t)$ represent different IMFs and final residual, respectively. Here, the number of IMFs is adaptively determined by the algorithm. Please refer to (Huang et al., 1998 and Mookah et al., 2015) for complete procedures. The final residual is a monotonic function or a local extremum.The IMF periods are calculated as follows: (a) estimate the auto-correlation sequence of each IMF, (b) find peaks separated by more than three elements and return their locations, (c) calculate the mean of the differences between adjacent elements.

\subsubsection{Description of EMD-MFDFA method}

The Multifractal Detrended Fluctuation Analysisproposed by Kantelhardt et al., (2002), is used in this paper to investigate the physical characteristics of the ISR data. The procedure can be described as follows:

Let $\left\{x_{t} \mid t=1,2, \ldots, N\right\}$ be an original time series of $N$ equidistant measurements to which the procedure of the EMD-MFDFA method is applied. It can be briefly described according to Lui et al. (2015), as:

Firstly, by decomposing the series $\left\{x_{t} \mid t=1,2, \ldots, N\right\}$ by EMD, one can obtain different order IMFs components and the final residue $r$. The parameter $r$ is the trend of the series. Then, by subtracting the trend part, the result becomes:

$$
y(t)=x_{t}-r
$$

Secondly, the profile $y(t)$ is then divided into 
$N=\operatorname{int}(N / s)$ equal-sized nonoverlapping windows with a length $s$. Since $N$ is not the integral multiple of $s$ in most cases, they might be a short part at the end of the profile that remains uncovered. To take full account of the series, the same procedure can be repeated starting from the end of the series. Hence, we obtain $2 N s$ segments all together.

Thirdly, as the trend of the series has been eliminated in Step 1, we do not need the least squares (LS) method fitting detrending step of the original MFDFA (Kantelhardt et al., 2002; Agbazo et al., 2019). One then calculates the $F^{2}(v, s)$ of each window as follows:

$$
\begin{aligned}
& F^{2}(v, s)= \\
& \left\{\begin{array}{c}
\frac{1}{s} \sum_{i=1}^{s}\left\{Y_{[(v-1) s+i]}\right\}^{2} \text { for } v=1,2, \ldots, N_{s} \\
\frac{1}{s} \sum_{i=1}^{s}\left\{Y_{\left[N-\left(v-N_{S}\right) s+i\right]}\right\}^{2} \text { for } v=\left(N_{s}+1\right), \ldots, 2 N_{s}
\end{array}\right.
\end{aligned}
$$

Fourthly, by averaging over all windows, one obtains the fluctuation as follows:

$$
F_{q}(s)=\left\{\begin{array}{l}
\left\{\frac{1}{2 N_{s}} \sum_{v=1}^{2 N_{s}}\left[F^{2}(v, s)\right]^{q / 2}\right\}^{1 / q} \text { for } q \neq 0 \\
\exp \left\{\frac{1}{4 N_{s}} \sum_{v=1}^{2 N_{s}} \ln \left[F^{2}(v, s)\right]\right\} \text { for } q=0
\end{array}\right.
$$

Finally, one analyzes the $\log -\log$ plots of $F_{q}(s)$ versus $s$ for each order of moment $q$ and determine the scalingbehavior of the fluctuation functions. According to (Kantelhardt et al., 2002), if the time series follow the power law, then we can obtain the scaling function:

$\mathrm{F}_{\mathrm{q}}(\mathrm{s}) \propto \mathrm{s}^{\mathrm{h}(\mathrm{q})}$

Where $h(q)$ is the generalized Hurst index function (Kantelhardt et al., 2002; Telesca et al., 2008, 2011). For monofractal time series, $h(q)$ is independent of $q$, whereas, for a multifractal time series, $h(q)$ varies with $q$. In order to avoid a divergence of moments in the fat tails of the fluctuation distribution as mentioned by some authors (Wang et al., 2013), we restrict the order $q$ within the range $-5 \leq q \leq 5$ as in (Wang et al., 2013).

\subsubsection{Multifractal Spectra (MFS)}

In order to get more information about ISR data and to characterize the strength of the multifractality, singularity spectrum $(f(\alpha))$ and the multifractal spectrum width $(\Delta \alpha)$ are determined. Indeed, according to Feder et al., (1988); Kantelhardt et al., 2002; Liu et al., 2014a, 2015; Agbazo et al., 2019), using Legendre transform, the relationship between $f(\alpha)$ and the generalized Hurst index $h(q)$ can be obtained and written as:

$\left\{\begin{array}{c}\alpha(q)=h(q)+q \frac{d h(q)}{d q} \\ f(\alpha)=q[\alpha(q)-h(q)]+1\end{array}\right.$

$\alpha(q)$ and $f(\alpha)$ are respectively singularity exponent (or Hölder exponent) and Singularity spectrum. The multifractal spectrum width is written as:

$\Delta \alpha=\alpha_{\max }-\alpha_{\min }$
Where $\alpha_{\max }$ and $\alpha_{\min }$ are obtained from the relation $f(\alpha)=0$.

$\Delta \alpha$ shows the probability of the difference between the maxima and minima. The bigger the multifractal strength is, the larger the value of $\Delta \alpha$ is also; therefore, $\Delta \alpha$ can be used to measure the complexity of sequences. In other words, if a spectrum has a broad width, $\Delta \alpha$ is indicative of a strong multifractality. If the width becomes smaller, then the time series tends to be a monofractal one (Liu et al., 2014a). In order to identify the IMF which can represent the original series, we have performed the MFFDA-MFS method on each IMF and the profile. The multifractal spectrum width, $\Delta \alpha$ of each IMF are compared to that of the profile.

\section{RESULTS AND DISCUSSION}

The original ISR time series and its IMFs are displayed in Figure 3. The ISR time series are not white noise because the total number of IMFs is different from $\log 2(N)$, where $N$ is the number of total data points. Figure 3 shows that the ISR can be decomposed into oscillatory intrinsic mode functions and the trend. We obtain twelve (12) IMFs and a residue that describe the local cyclical variability of the original signal. The residue represents the original signal at different timescales mainly the long-term trend for the data. One can note that polynomial trend exists in the ISR time series from the trend. However, the order of the polynomial, which can be used to fit the local trend cannot be chosen arbitrarily. It seems that quadratic-order polynomial may be adopted. It is important to keep in mind that the order of the polynomial may vary with the length of the study period. In this study only the data recorded for two years are used. The observed trend shows a small decrease over the data set.
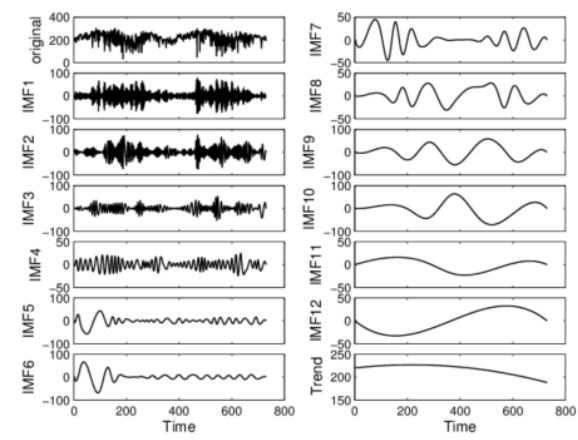

Fig.3: Empirical Mode Decomposition of the ISR in twelve (12) intrinsic mode functions (IMFs) and a trend

Table 1: Period of the IMFs obtained from the ISR data set.

\begin{tabular}{|c|c|c|c|c|c|c|}
\hline IMF & 1 & 2 & 3 & 4 & 5 & 6 \\
\hline $\begin{array}{c}\text { Period } \\
\text { (days) }\end{array}$ & 5.6 & 9.8 & 15.2 & 37.3 & 54 \\
\hline IMF & 7 & 8 & 9 & 10 & 11 & 12 \\
\hline $\begin{array}{c}\text { Period } \\
\text { (days) }\end{array}$ & 83 & 109 & 182 & 243 & 532 & 728 \\
\hline
\end{tabular}


The IMFs periods are reported in Table 1. The fastest component has a cycle of 2.9 days, contrasting the slowest cycle of 728 days. Let's notice that the first four (4) IMFs represent the inter-day cycles, the next six (6) IMFs (IMF 5-10) represent the inter-month cycles while the remaining IMFs 10-12 are associated with the intrayear cycles representing longer periods and may (or may not) represent actual physical processes. The fact that the EMD technique decomposes the incoming shortwave radiation time series into a set of physically meaningful modes (without any necessity to define a basis function) gives us confidence that the technique is of significant utility.

The plot of the generalized Hurst exponent $h(q)$ versus the moment order $q$ obtained from each IMF times series are shown in Figure 4. From the IMFs series, $h(q)$ values of different orders $q$ is in descending order instead of being constant. Thus, these series comprise multifractal characteristics, which cannot be described fully using traditional monofractal theory. However, the exponents $h(q)$ change little with the increase value of $q$ in some IMFs, indicating the weak multifractal behavior. To be specific, all the $h(2)$ values, known as Hurst exponent (see Kantelhardt et al., 2002), are lower than 0.5. One can conclude that the IMFs series are nonstationary signals with long range power-law correlations. Therefore, the fluctuations of the IMFs time series are negatively correlated in a power-law fashion.

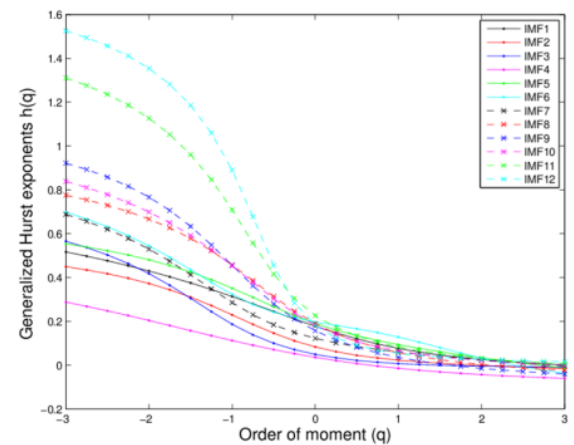

Fig.4: Generalized Hurst index $h(q)$ versus q relationship for each Intrinsic mode functions (IMFs)

In order to get more information about the multifractality of each IMFs, the corresponding multifractal spectrum $f(\alpha)$ is performed. In Figure 5, the singularity spectra are presented as a function of the singularity exponent $(\alpha)$ foreach IMFs series. It is obvious that the shape of $f(\alpha)$ curves for IMFs are like hooks to the right. These different shapes reflect inner dynamical characteristics of IMFs series. In all cases, the spectrum shape is a humped curve, that has a left truncation and it is obviously unsymmetrical about its axis. This is attributed to multifractal structure that is insensitive to the local fluctuations with large magnitudes. Meanwhile, it also shows that multifractal characteristics of IMFs change from one IMF to another.

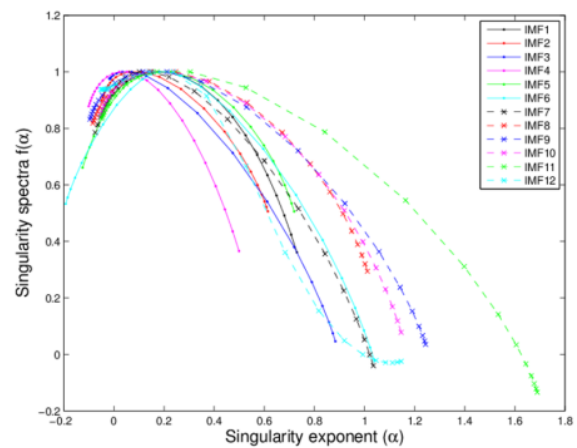

Fig.5: Singularity spectrum $f(\alpha)$ versus singularity exponent $\Delta \alpha$

In order to assess the strength of the multifractality, multifractal spectrum width $\Delta \alpha$ obtained for the detrended original series (D-ori) and each intrinsic mode functions (IMFs) are presented in Figure 6.

Figure 6 reveals that spectrum width $\Delta \alpha$ varies from one IMFs to another. Moreover, the IMFs have different multifractals behaviors comparatively to the original one. So, none of the IMFs can represent the original time series. There is no relationships between $\Delta \alpha$ values and the IMFsperiods. Therefore, the strength of their multifractality does not depend on the period of oscillation. Theseresultsimply a stochastic dynamic character and strong fluctuations of the IMFs series.

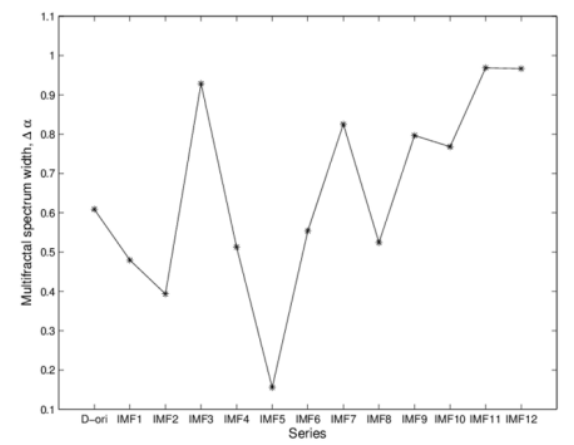

Fig.6: Multifractal spectrum width $\Delta \alpha$ obtained for detrended series (D-ori) and each intrinsic mode functions (IMFs)

\section{CONCLUDING REMARKS}

In this paper, incoming shortwave radiation (ISR) time series measured in 2008-2009 period at Nalohou AMMA-CATCH station is decomposed in oscillatory Intrinsic Mode Functions (IMFs) and its fractal characteristics are analyzed. Each IMF is guessed to be the effect of a different physical cause with different characteristic time. The decomposition is done by Empirical Mode Decomposition (EMD). The fractals characteristics of IMF are studied by Multifractal Detrended Fluctuation Analysis (MFDFA) and Multifractal Spectra (MFS) method. The main and nontrivial findings obtained are the followings: (1) the original incoming shortwave radiation (ISR) time series is not white noise because the total number of IMFs is different from $\log 2(N)$ where $N$ represents the number of 
total data points, therefore ISR time series can be predicted; (2) for the study period, ISR time series can be decomposed into twelve (12) oscillatory Intrinsic Mode Functions (IMFs) and the obtained trend presents decreasing behavior; (3) one can note that polynomial trend exists in the incoming shortwave radiation time series seeming that quadratic-order polynomial may be adopted; (4) all the IMFs exhibit distinct multifractal behaviour which strength depends strongly on the IMFs; (5) the strength of the IMF multifractality cannot be predicted according to the increasing of its period.

It's will be important to have a large set of ISR data for improving the study. For this end, a Detrended Cross Correlation Analysis (DCCA) method can be prospected and used for filling the gaps in the ISR data at Nalohou. This method will allow us to obtain an extended period of complete set of ISR. That will be the object of future studies.

\section{Conflicts of Interest}

The authors declare no conflicts of interest in this paper.

\section{Acknowledgments}

The authors would like to thank the Supervisors of the AMMA-CATCH data base for providing the data.

\section{References}

Agbazo, M. N., Koto N'Gobi, G., Alamou, E., Kounouhewa, B., Afouda, A., 2019. Fractal Analysis of the Long-Term Memory in Precipitation over Benin (West Africa). Hindawi, Advances in Meteorology, Volume 2019, Article ID 1353195, 12 pages, https://doi.org/10.1155/2019/1353195, 2019.

Behrang, M.A., Assareh, E., Ghanbarzadeh, A., Noghrehabadi, A. R., 2010. The potential of different Artificial Neural Network (ANN) techniques in daily global solar radiation modeling based on meteorological data. Solar Energy 84 (8), 1468-1480.

Berger, B. S., Minis, I., Rokni, M., Papadopoulos, M., Deng, K., Chavalli, A., 1997 Cutting state identification. J. Sound Vib. 1997, 200, 15-29.

Bengulescu, M., Blanc, P., Wald, L., 2016.Characterizing temporal variability in measurements of surface solar radiation and its dependence on climate. Energy Procedia 97, 164-171. https://doi.org/10.1016/ j.egypro.2016.10.045.

Calif, R., Schmitt, G., Huang, Y., Soubdhan, T., 2013 Intermittency study of high frequency global solar radiation sequences under a tropical climate. Sol. Energy 98, 349-365. https://doi.org/10.1016/j.solener. 2013.09.018.

Chu, Y., 2011. Review and Comparison of Different Solar Energy Technologies. Res. Assoc. Glob. Energy Netw. Inst. 619, 595-0139.

Flandrin, P., Gonçalvès, P., 2004. Empirical mode decomposition as data-driven wavelet-like expansions, International Journal of Wavelets, Multiresolution and Information Processing, 2(4), 477-496.

Hocaoglu, F.-O., Gerek, O.-M.-N., Kurban, M., 2008. Hourly solar radiation forecasting using optimal coefficient 2-D linear filters and feed-forward neural networks. Solar Energy 82 (8), 714-726.

Huang, N. E., Shen, S. S., 2005. Hilbert-Huang Transform and Its Applications. World Scientific Publishing Company: Singapore.

Huang, N., Wu, M., Long, S., Shen, S., Qu, W., Gloersen P., Fan, K., 2003. A confidence limit for the empirical mode decomposition and Hilbert spectral analysis, Proc. R. Soc. London, 459, 2317-2345.

Huang N. E., Wu, M.C., Long, S. R., Shen, S. S. P., 2003. A confidence limit for the empirical mode decomposition and Hilbert spectral.

Huang, N. E., Shen, Z., Long, S. R., Wu, M. C., 1998. The empirical mode decomposition and the Hilbert spectrum for nonlinear and non-stationary time series analysis. Proc. Math. Phys. Eng. Sci., 454, 903-995.

Kantelhardt, J. W., Zschiegner, S. A., Koscielny-Bunde, E., 2002. Stanley H. E. Multifractal detrended fluctuation analysis of nonstationary time series. Physica A: Statistical Mechanics and Its Applications, vol. 316, no. 1-4, pp. 87-114.

Lele, I., et al., 2016. Variability of the Intertropical Front (ITF) and Rainfall over the West African Sudan-Sahel Zone. J. Clim. https://doi.org/10.1175/2010J CLI3277.1.

Liu, Z. H., Xu, J. H., Chen, Z. S., 2014a. Multifractal and long-term memory of humidity processing the Tarim River basin. Stoch. Environ. Res. Risk Assess. 28, 1383-1400.

Liu, Z., Xu, J., Shi, K., 2014. Self-organized criticality of climate change. Hinadv in meteo 685-691.

Mamadou, O., Cohard, J-M., Galle, S., Awanou, C. N., Diedhiou, A., Kounouhewa, B., Peugeot, C., 2014 Energy fluxes and surface characteristics over a cultivated area in Benin: daily and seasonal dynamics 893-914.

Mandelbrot, B.B., 1982. The Fractal Geometry of Nature. W. H. Freeman and Co., New York.

Mookiah, M. R. K., Achary, U. R., 2015. Automated detection of age-related macular degeneration using empirical mode decomposition. Knowl. Based Syst, 89, 654-668.

Mellit, A., Benghanem, M., Arab, A.H., Guessoum, A. A., 2005.Simplified model for generating sequences of global solar radiation data for isolated sites using artificial neural network and a library of Markov transition matrices approach. Solar Energy 79 (5), 469-482. 
Sang, Y. F., Wang, Z., Liu, C., 2012. Period identification in hydrologic time series using empirical mode decomposition and maximum entropy spectral analysis. J. Hydrol. 2012, 424-425, 154-164.

Telesca, L., Bernardi, M., Rovelli, C., 2008. Time-scaling analysis of lightning in Italy. Communications in Nonlinear Science and Numerical Simulation, 13(2008) 1384-1396. Science Direct: www.elsevier.com/locate/cnsns.

Telesca L, Lovallo M. Analysis of the time dynamics in wind records by means of multifractal detrended fluctuation analysis and the Fisher-Shannon information plane. J Stat Mech. 2011: P07001, 2011.

Wang, J., Shang, P., Dong, K., 2013. Effect of linear and nonlinear filters on multifractal analysis. Appl. Math. Comput.224,337-345. https://doi.org/10.1016/j.amc. 2013.08.037.

Wu, Z., Huang, N., 2003. A study of the characteristics of white noise using the empirical mode decomposition method, Proc. R. Soc. London, 460, 1597-1611.

Zeng, Z., Yang, H., Zhao, R., Meng, J., 2013. Nonlinear characteristics of observed solar radiation data. Sol. Energy 87, 204-218.

Zhang, X., Qi, Y., Zhu, M., 2014. Characteristic Analysis of White Gaussian Noise in S-Transformation Domain. J. Comput. Commun. 2014, 2, 20-24. 